\title{
New processing approach and results for bistatic TerraSAR-X/F-SAR spaceborne-airborne experiments
}

\author{
Marc Rodriguez-Cassola, Pau Prats, Stefan V. Baumgartner, Gerhard Krieger, \\ Anton Nottensteiner, Ralf Horn, Irena Hajnsek, Alberto Moreira \\ *Microwaves and Radar Institute, German Aerospace Center (DLR), 82234 Wessling, Germany \\ Phone/Fax: +498153282392/1449, Email: marc.rodriguez@dlr.de
}

\begin{abstract}
Following the success of the first bistatic spaceborne-airborne experiment between TerraSAR-X and FSAR carried out in November 2007, DLR has performed a second bistatic experiment in July 2008 with new challenging acquisitions. Furthermore, the existing bistatic processing chain has been updated with two significant improvements: a) clock offset synchronisation is now performed without the use of reference targets, and b) SAR imaging is done using a fast focussing technique. The new SAR imaging algorithm, based on the fast factorised backprojection algorithm, has proved very good focussing qualities while dramatically reducing (up to a factor 100 with respect to direct backprojection) the overall computational load. The new processing chain is tested using the image of the first TerraSAR-X experiment. Results of a dualpol acquisition performed during the second TerraSAR-X/F-SAR experiment and showing the first dual-pol bistatic spaceborneairborne images are also presented in this paper.
\end{abstract}

\section{INTRODUCTION}

Early November 2007, DLR carried out the first X-band spaceborne-airborne SAR experiment, using TerraSAR-X as transmitter and DLR's new airborne SAR, F-SAR, as receiver. This experiment was the first of its kind in Europe, and the first to demonstrate high-resolution bistatic imaging for such configurations [1]-[3]. Fig. 1 depicts the bistatic configuration flown during this experiment. Early July 2008, DLR conducted a second spaceborne-airborne experiment for testing new challenging acquisitions. Among these, a dual-pol bistatic experiment was carried out, showing the potentials of future polarimetric bistatic acquisitions.

In the framework of DLR bistatic SAR activities, a new bistatic proceesing approach has been developed to help overcome the main limitations of the one used for previous experiments [2]-[4]. The new processing chain offers two significant advantages with respect to its predecessor: a) no reference targets are needed for clock offset syncrhonisation, and $b$ ) precise fast bistatic SAR imaging is now available. The former allows first-order time and Doppler syncrhonisation to be performed automatically, since no position information is needed in the point-like target analysis. The latter, based on the fast factorised backprojection algorithm [5], and thus named bistatic fast factoriesd backprojection algorithm (BFFBP), has been newly developed to accommodate the particularities of general bistatic SAR configurations. Processing time improvements of about 100 with respect to direct backprojection have been obtained in the processing of the available bistatic data sets. An image of the first experiment processed with the new

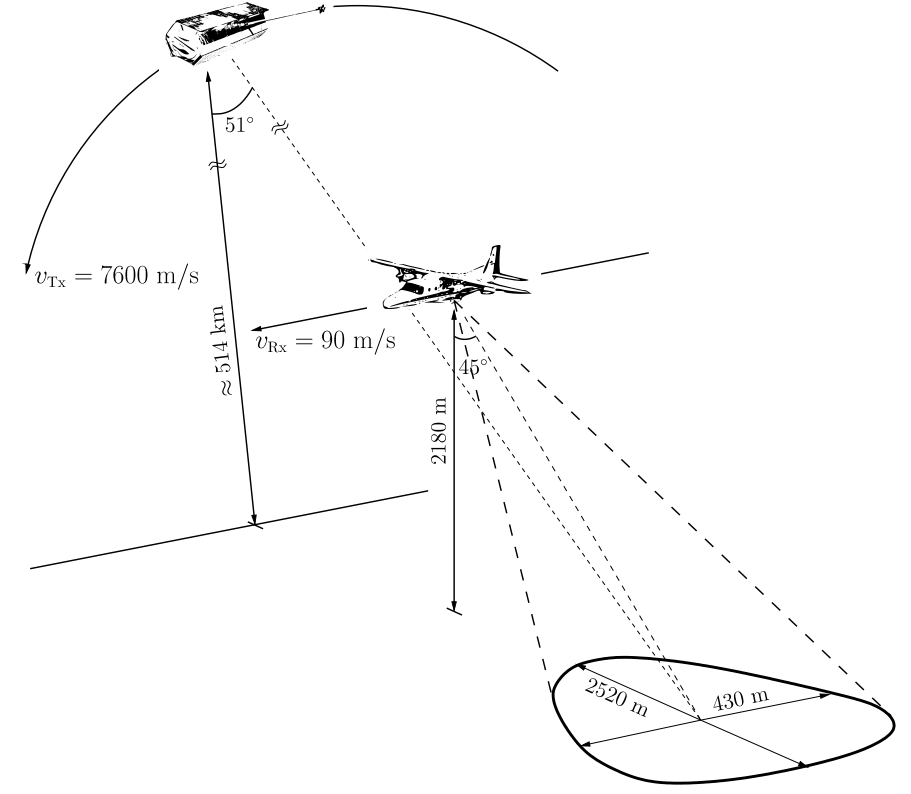

Fig. 1. Bistatic spaceborne-airborne configuration used in first DLR TerraSARX/F-SAR experiments.

chain and results of the dual-pol bistatic acquisitions are shown in the paper.

\section{NON-SYNCRHONISED SHORT-TIME BISTATIC ACQUISITIONS}

\section{A. Processing approach}

The general block diagram of a bistatic SAR processor must follow the structure of Fig. 2. The block diagram shows a two-step approach: a) range-Doppler synchronisation, and b) bistatic SAR imaging. All a priori information on the relative clock behaviour has to be introduced in the first range/Doppler synchronisation of the bistatic data set. This information is used for correcting both bistatic range time and the azimuth phase of the bistatic data. Only then bistatic SAR imaging is performed. If, after bistatic SAR imaging, residual phase errors can still be detected in the image, the updated residual phase errors can be reinjected in the synchronisation module followed by data reprocessing. In order to allow an accurate residual phase estimation, it is essential that no additional phase errors are introduced in SAR imaging, since this effect 


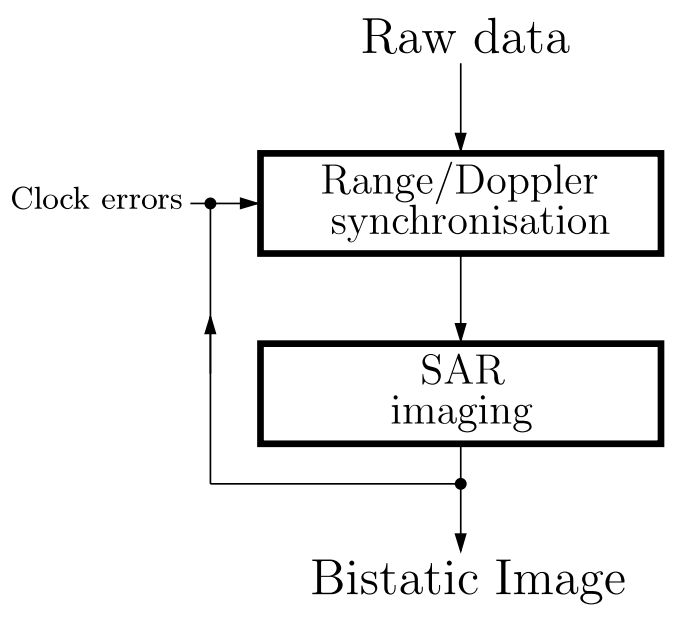

Fig. 2. Block diagram of a generalised bistatic SAR processor.

might deteriorate the results of the residual clock offset estimation procedure. In the case of non-synchronised bistatic radars, no a priori information on the clocks is available, thus all synchronisation information must be estimated from the data.

\section{B. Clock error model}

The clock error model for non-synchronised short-time bistatic acquisitions can be approximated by a constat frequency offset and a random phase error

$$
\phi_{\text {clock }}(t)=2 \pi \cdot \Delta f \cdot t+\phi_{\text {res }}(t)
$$

where $t$ denotes the general time axis. The short-time characteristics of the acquisition are denoted by the use of a constant frequency offset clock error. Depending on the clocks quality and the integration time of the synthetic aperture, $\phi_{\text {res }}(t)$ will have an increasing impact on the focussed image. A discussion on the clock error model can be found in [6], [7].

\section{Automatic range and Doppler synchronisation}

Point-like target analysis is needed for automatic synchronisation. However, unlike in the case of [3], [7], no absolute position of the targets is used for synchronisation. A good estimation and correction of $\Delta f$ might be sufficient for highresolution imaging purposes.

1) Clock offset estimation: A point-like target in the range compressed data with good SNR is needed for the clock offset estimation. Clock offset is estimated by matching both phase history and range migration components of this single point-like target, considering that range error is proportional to $\Delta f / f_{0, \mathrm{Tx}}$ and phase error is directly proportional to $\Delta f$.

2) Residual phase error estimation: Additional phase information is needed for estimating residual phase errors. If the topography information of the scene is known, then a least-mean-squares estimate of the residual phase error can be extracted using an autofocus algorithm.

\section{BISTATIC FAST-FACTORISED BACKPROJECTION}

Due to the complexity of simultaneously achieving precise bistatic imaging and small computational load using Fourierdomain techniques, a new processing algorithm based on fast factorised backprojection (FFBP) [5] has been developed. The new imaging algorithm, named bistatic fast factorised backprojection (BFFBP), achieves precise accommodation of azimuthvariance and topography of general bistatic SAR acquisitions, while keeping a speed-up factor proportional to $\log _{2} N$ with respect to direct backprojection (DBP), in a similar manner as Fourier-domain algorithms do.

\section{A. Image reference system}

The reference system for subimage computations has an elliptical range coordinate and an angular coordinate referenced to the transmitter trajectory (but might as well be defined to the receiver's, if convenient). This reference system, shown in Fig. 3 in the general case, keeps the advantage of lowest bandwidth images while offering an advantageous manner of displaying topography information.

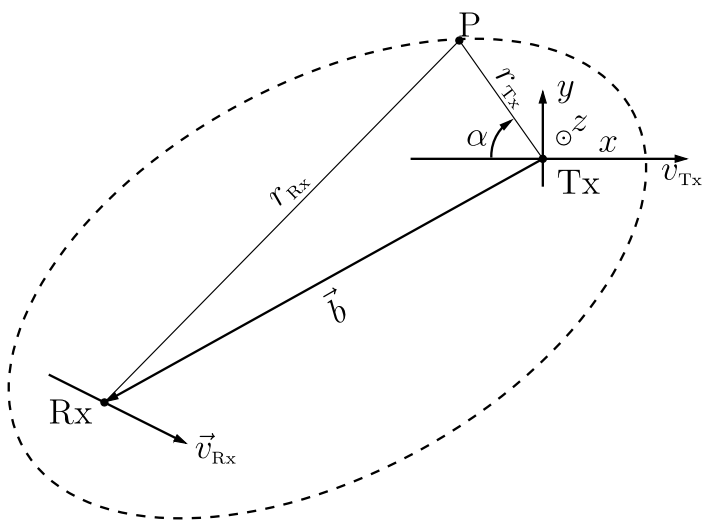

Fig. 3. Reference grid used in BFFBP. $\mathrm{P}$ is the target whose coordinates in the elliptical grid are $\left(r_{\mathrm{Tx}}+r_{\mathrm{Rx}}, \alpha\right)$.

\section{B. Nyquist requirements}

The Nyquist angular requirements for general along-track bistatic acquisitions can be shown to be

$$
\delta(\cos \alpha) \geq \frac{\lambda}{T \cdot r_{\mathrm{Tx}} \cdot\left(\omega_{\mathrm{Tx}}+\omega_{\mathrm{Rx}}\right)},
$$

where $\lambda$ is the radar wavelength, $T$ is the duration of the synthetic aperture, $r_{\mathrm{Tx}}$ is the range transmitter-target, and $\omega_{\mathrm{Tx}}, \omega_{\mathrm{Rx}}$ are transmitter's and receiver's instantaneous radial velocities, respectively. The non-constant value of this Nyquist requirement is due to the space-variant character of bistatic SAR.

\section{Implementation}

BFFBP is implemented in a similar manner to FFBP [5], but accounting for the modifications in the reference system and the Nyquist requirements of the previous subsections. The algorithms is tested using actual data of the first bistatic 
TerraSAR-X/F-SAR experiment. Results are shown in section IV.

\section{DLR BISTATIC SAR EXPERIMENTS TERRASAR-X/F-SAR}

\section{A. First experiment TerraSAR-X/F-SAR}

A complete overview on the first experiment TerraSARX/F-SAR, performed early November 2007, can be found in [1]-[3]. The bistatic image obtained using the new bistatic processing chain is shown in Fig. 4. A speed-up factor of about

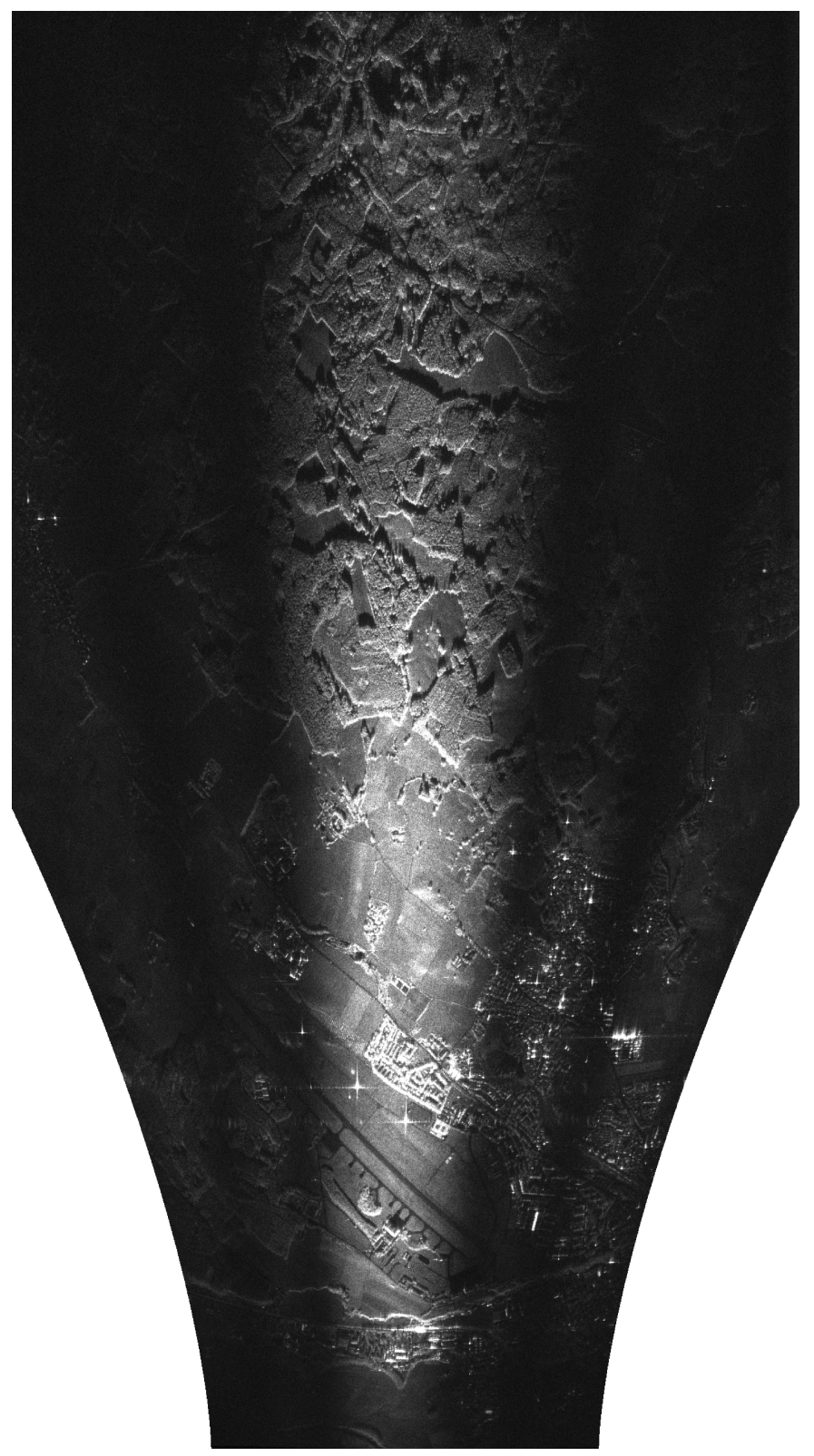

Fig. 4. BFFBP-processed bistatic image of the TerraSAR-X/F-SAR first spaceborne-airborne experiment. Radar illumination from the bottom.

100 has been achieved by using the new processing approach, especially due to the use of BFFBP.
TABLE I

PARAMETERS OF BISTATIC DUAL-POL EXPERIMENT

\begin{tabular}{lc}
\hline Acquisition mode & Spotlight Dual-pol \\
Integration time [s] & 2.56 \\
Pulse repetition frequency [Hz] & 6407 \\
Wavelength [m] & 0.031 \\
Transmitted bandwidth [MHz] & 100 \\
Sampling frequency [MHz] & 125 \\
TerraSAR-X effective velocity [m/s] & 7408 \\
F-SAR nominal velocity [m/s] & 90 \\
TerraSAR-X altitude $[\mathrm{km}]$ & 514 \\
F-SAR altitude [m] & 2180 \\
\hline
\end{tabular}

\section{B. Dual-pol experiment TerraSAR-X/F-SAR}

The second experiment TerraSAR-X/F-SAR was performed early July 2008 and included a dual-pol bistatic acquisition. TerraSAR-X was toggled to transmit alternately up- and downchirps with $\mathrm{H}$ and $\mathrm{V}$ polarisations, respectively. F-SAR received the data with an vertically-polarised antenna, thus allowing retrieval of $\mathrm{HV}$ and VV bistatic components. The configuration and the acquisition were similar to those of the first experiment, but some of the acquisition parameters changed. Table I summarises the bistatic dual-pol acquisition.

Fig. 5 and 6, respectively, show the bistatic copol (VV) and crosspol (HV) images acquired during the second TerraSAR$\mathrm{X} / \mathrm{F}-\mathrm{SAR}$ bistatic spaceborne-airborne experiment. As expected, SNR is significantly better in the copol image. Noticeable differences, above all in distributed targets, between both images have been observed. Like in the case of the image obtained in the first TerraSAR-X/F-SAR experiment, the integrated F-SAR antenna pattern is clearly visible in the processed image.

\section{Summary}

A new processing approach for non-synchronised shorttime bistatic acquisitions has been presented, including synchronisation steps without reference targets and a fast and precise bistatic fast-factorised backprojection (BFFBP) imaging algorithm. The new processing chain has been tested with actual data of the first TerraSAR-X/F-SAR experiment (November 2007). Results of the dual-pol acquisition of the second TerraSAR-X/F-SAR experiment (July 2008) are also presented.

\section{REFERENCES}

[1] S. V. Baumgartner, M. Rodriguez-Cassola, A. Nottensteiner, R. Horn, M. Schwerdt, R. Scheiber, U. Steinbrecher, R. Metzig, M. Limbach, J. Mittermayer, G. Krieger, A. Moreira, Bistatic experiment using TerraSAR-X and DLR's new F-SAR system, Proc. EUSAR 2008, Friedrichshafen, Germany.

[2] M. Rodriguez-Cassola, S. V. Baumgartner, A. Nottensteiner, R. Horn, M. Schwerdt, P. Prats, J. Fischer, U. Steinbrecher, R. Metzig, M. Limbach, G. Krieger, A. Moreira, Bistatic spaceborne-airborne experiment TerraSAR-X/F-SAR: data processing and results, Proc. IGARSS 2008, Boston, USA.

[3] M. Rodriguez-Cassola, S. V. Baumgartner, G. Krieger, A. Moreira, Bistatic TerraSAR-X/F-SAR spaceborne-airborne SAR experiment: description, data processing and results, submitted to IEEE Trans. Geosci. Remote Sens.Jul. 2004. 


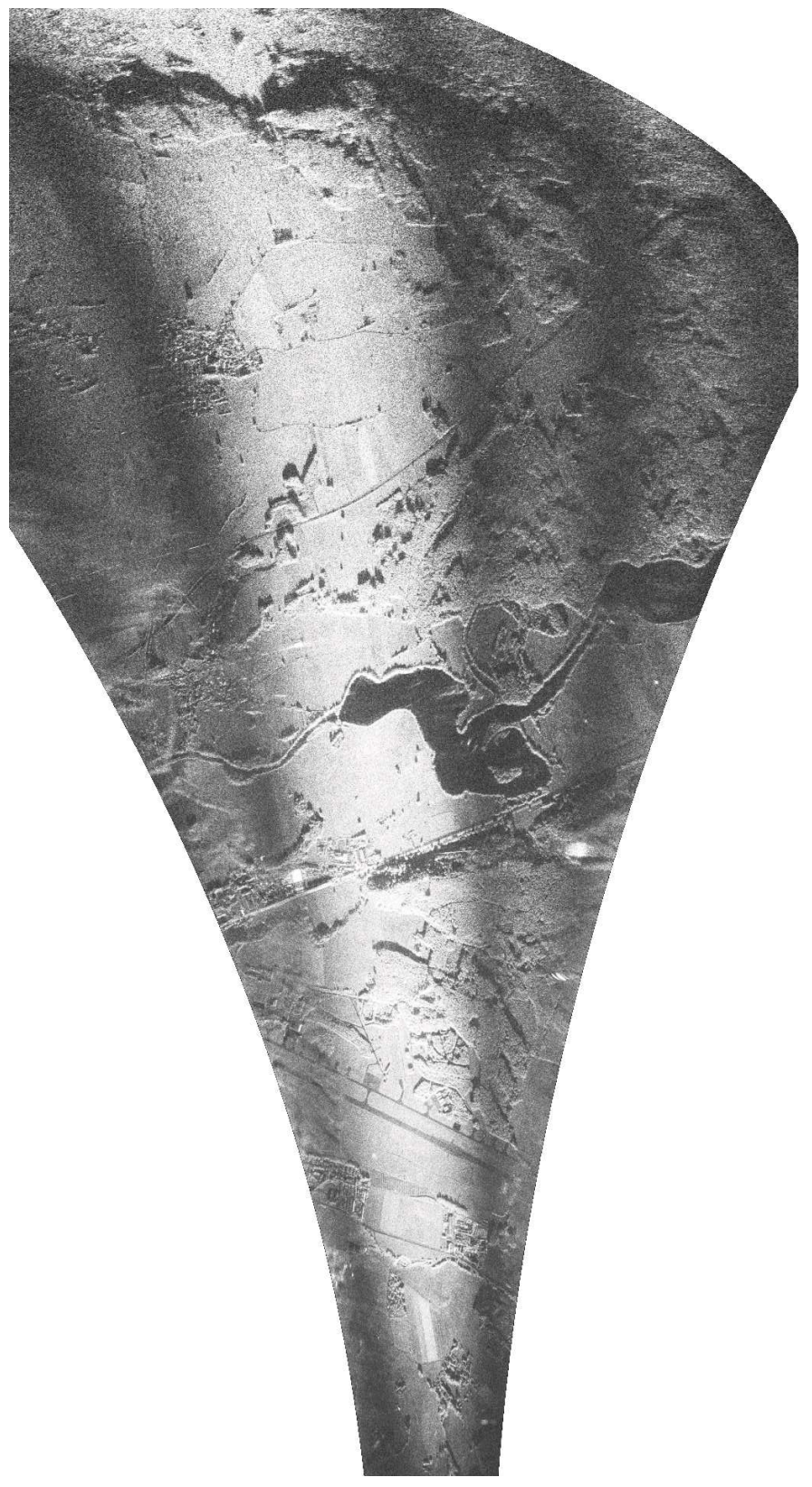

Fig. 5. Bistatic copol (VV) image of the bistatic TSX/F-SAR dual-pol experiment. Radar illumination from the bottom.

[4] H. Cantalloube, M. Wendler, V. Giroux, P. Dubois-Fernández, G. Krieger Challenges in SAR Processing for Airborne Bistatic Acquisitions, Proc. EUSAR 2004, pp. 577-580, Ulm, Germany.

[5] L. Ulander, H. Hellsten, G. Stentström, 'Synthetic-aperture radar processing using fast factorized back-projection', IEEE Trans. Aerosp. Electr Syst., vol. 39, no. 3, pp. 760-776, Jul. 2003.

[6] G. Krieger, M. Younis, Impact of Oscillator Noise in Bistatic and Multistatic SAR, IEEE GRSL 3-3, 2006.

[7] P. Lopez-Dekker, J. J. Mallorqui, P. Serra-Morales, and J. Sanz-Marcos, "Phase Synchronization and Doppler Centroid Estimation in Fixed Receiver Bistatic SAR Systems", IEEE Trans. Geosci. Remote Sens., vol. 46, no. 11, pp. 3459-3471, Nov. 2008.

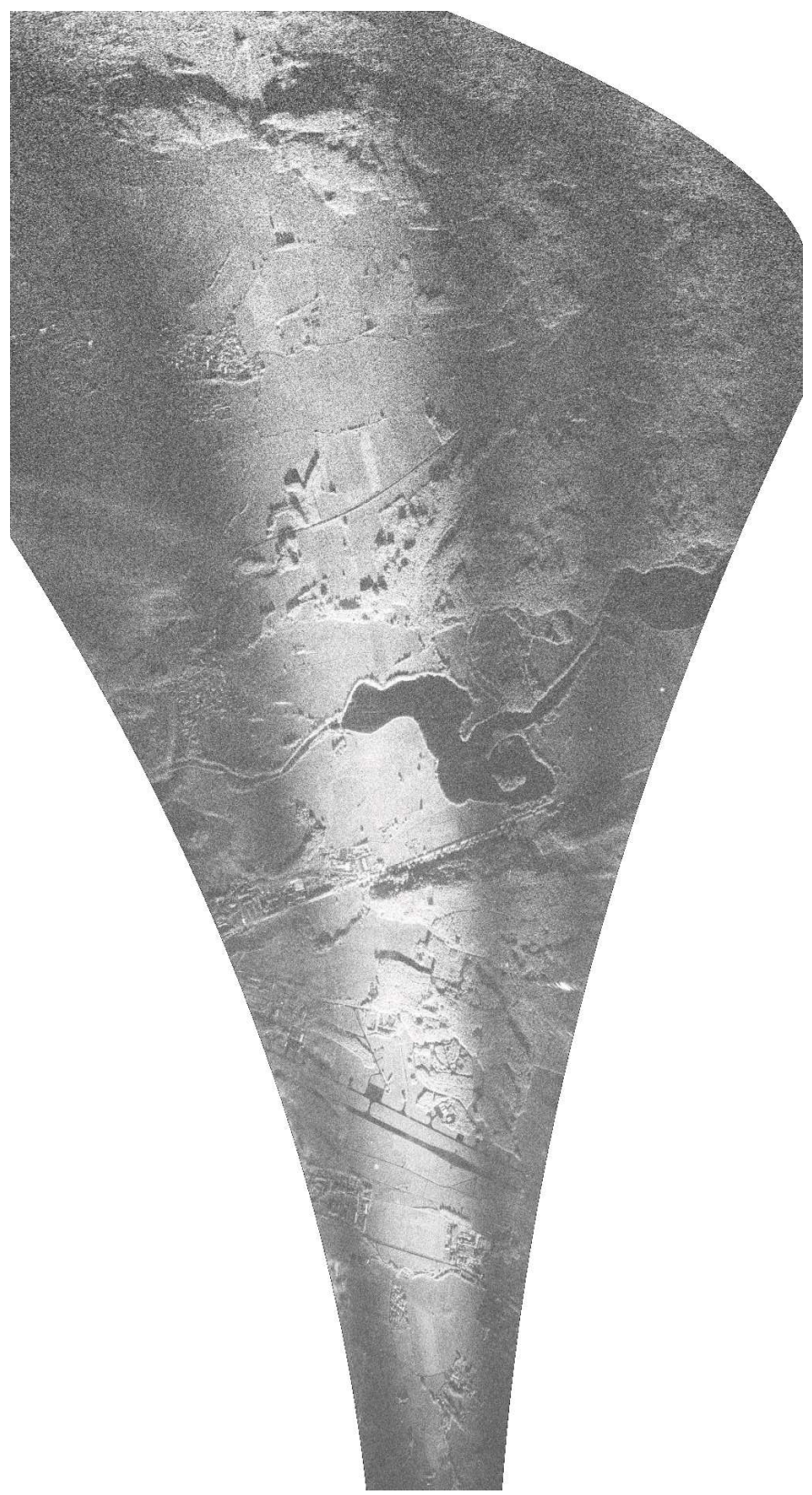

Fig. 6. Bistatic crosspol (HV) image of the bistatic TSX/F-SAR dual-pol experiment. Radar illumination from the bottom. 